\title{
Mechanism of the inhibitory effect of ghrelin in sepsis
}

This article was published in the following Dove Press journal:

Hepatic Medicine: Evidence and Research

24 February 2010

Number of times this article has been viewed

\section{Asha Jacob \\ Rongqian Wu \\ Mian Zhou \\ Gene F Coppa \\ Ping Wang}

Department of Surgery, North Shore University Hospital and Long Island Jewish Medical Center, and

The Feinstein Institute for Medical Research, Manhasset, NY, USA
Correspondence: Ping Wang Laboratory of Surgical Research, The Feinstein Institute for Medical Research, 350 Community Drive, Manhasset, NY I 1030, USA

Tel + I $516562-34||$

Fax +I 516 562-1022

Email pwang@nshs.edu

\begin{abstract}
Sepsis and septic shock are the leading causes of death in intensive care units. Approximately $40 \%-70 \%$ of the mortality is associated with severe sepsis and septic shock. Systemic antibiotic usage, surgical intervention, aggressive fluid resuscitation and careful monitoring are common measures currently used to treat sepsis. Despite the advances in the understanding of the pathophysiology of sepsis, very little progress has been made towards therapeutic interventions. Recently we have shown that ghrelin, a stomach-derived peptide which is an endogenous ligand for the growth hormone secretagogue receptor (GHSR-1a), is beneficial in attenuating the inflammatory response, organ injury and mortality in an experimental model of polymicrobial sepsis induced by cecal ligation and puncture (CLP). In this review, we describe the mechanism of action of ghrelin in sepsis, highlight the role ghrelin plays in attenuating the hepatic dysfunction induced by sepsis and septic shock and suggest in developing ghrelin as a potential therapy for sepsis.
\end{abstract}

Keywords: ghrelin, sepsis, inhibition septic shock, GHSR-1a, cecal ligation

\section{Introduction}

Sepsis and septic shock continue to be common causes of death in intensive care units. Septic shock and multi-organ dysfunction are the predominant causes of death in patients with sepsis. The incidence of severe sepsis in the United States is estimated to be 750,000 cases per year and continues to increase at a rate of $1.5 \%$ annually. ${ }^{1}$ Approximately $40 \%-70 \%$ of the mortality rate is associated with severe sepsis and septic shock. ${ }^{2}$ Despite advances in the understanding of the pathophysiology of sepsis, very little improvement has been made in therapeutic interventions. In this review, we provide a brief overview of the pathophysiology of sepsis as it relates to hepatic dysfunction and describe the preclinical studies detailing the mechanism of action of ghrelin, a novel stomach-derived peptide that could be developed as a potential therapy for sepsis.

\section{Hepatic dysfunction in sepsis}

Due to its integral role in metabolism and host defense mechanisms, liver is believed to be the major organ responsible for initiating multiple organ failure during sepsis. ${ }^{3}$ The cardiovascular responses to polymicrobial sepsis induced by cecal ligation and puncture (CLP) in rats is characterized by an early hyperdynamic phase (2-5 hours after CLP) associated with the increased cardiac output and decreased vascular resistance followed by a late phase (20 hours after CLP) which involved the decreased cardiac output and increased vascular resistance. ${ }^{3}$ Previous studies have indicated that hepatic function is depressed early after the onset of sepsis during the hyperdynamic response phase. ${ }^{4}$ 
This hepatic dysfunction is a consequence of the upregulated pro-inflammatory cytokine production by the Kupffer cells, the resident macrophages of the liver. ${ }^{5-7}$

Upregulation of cytokines is, in part, caused by the increased release of the sympathetic neurotransmitter, norepinephrine (NE) from the gut during sepsis. This is evidenced by our studies ${ }^{8}$ and others ${ }^{9}$ which show that peripheral sympathetic activity increases during sepsis resulting in the elevation of plasma levels of NE. In addition, enterectomy prior to onset of sepsis markedly reduced circulating levels of NE. ${ }^{10}$ This indicates that the gut is the major source of NE release during sepsis. About $50 \%$ of the NE released in the body is produced by the sympathetic fibers in the gut. ${ }^{11,12}$ Interestingly, NE levels in the portal blood during sepsis are significantly higher than those in the systemic circulation. Intraportal infusion of $\mathrm{NE}$ in vivo increased $\mathrm{TNF}-\alpha$ release and was inhibited by co-infusion with yohimbine, a nonspecific antagonist of the $\alpha_{2}$-adrenoceptor. ${ }^{13}$ Cellular levels of TNF- $\alpha$ in Kupffer cells were also significantly increased following intraportal NE infusion and were inhibited by co-infusion with yohimbine.

The increase in TNF- $\alpha$ release was abrogated by coadministration of intraportal infusion of NE and the specific $\alpha_{2 \mathrm{~A}}$ antagonist, BRL-4408 maleate. ${ }^{14}$ Kupffer cells isolated from rats during early sepsis ( 2 hours after CLP) exhibited marked increase in $\alpha_{2 \mathrm{~A}}$-adrenoceptor mRNA expression. ${ }^{14}$ Recently, we showed that mitogen activated protein kinase (MAPK) phospatase-1 (MKP-1) is significantly decreased in the liver during sepsis and this decrease is correlated at least in in vitro, by the activation of NE on Kupffer cells. ${ }^{15}$ In addition, intraportal infusion with $\mathrm{NE}$ in vivo in normal rats significantly decreased MKP-1 mRNA expression. ${ }^{15}$ These studies collectively suggest that NE released from the gut during the early stage of sepsis travels through the portal vein, binds to $\alpha_{2 \mathrm{~A}}$-adrenoceptor on Kupffer cells, possibly down regulates MKP-1 leading to uncontrolled activation of MAPKs, and facilitates the increased production of TNF- $\alpha$ thereby causes hepatic dysfunction (Figure 1).

\section{Role of ghrelin in sepsis}

Ghrelin was first identified from the rat stomach about a decade ago $^{16}$ and has demonstrated to be a strong stimulator of growth hormone secretion in the rat and human. ${ }^{17-19}$ Ghrelin mediates its effects by binding to the growth hormone secretagogue receptor-1a (GHSR-1a) and it is an endogenous ligand for this receptor. It has been also regarded as a potent vasodilatory peptide. ${ }^{20}$ Intravenous administration of ghrelin in healthy human volunteers produced significant reduction in peripheral vascular resistance and increased cardiac output without any significant change in heart rate. ${ }^{21}$ These cardiovascular responses mimic the hyperdynamic phase that is generally observed in the early stage of sepsis. ${ }^{3}$ The primary challenge in sepsis, however, is to prevent the transition from the early hyperdynamic phase to the late hypodynamic phase.

Since ghrelin has vasodilatory properties, we sought to determine whether ghrelin or its receptor, GHSR-1a, plays any role in the cardiovascular response to sepsis. Our study showed that while ghrelin levels decreased both at 5 hours and 20 hours after CLP, GHSR-1a mRNA expression was markedly elevated in early sepsis. Ghrelin-induced relaxation was also increased significantly during early sepsis but was not altered in late sepsis. These results suggest GHSR-1a expression is upregulated and vascular sensitivity to ghrelin stimulation is increased in the hyperdynamic phase of sepsis. ${ }^{22}$ Others have also shown that plasma ghrelin levels were significantly decreased in an animal model of endotoxemia as well. ${ }^{23}$ Therefore, these studies imply that ghrelin could be used as a treatment for sepsis.

Ghrelin has been reported to downregulate cytokines in human endothelial cells. ${ }^{24}$ However, the major source of inflammatory cytokines in sepsis are macrophages. ${ }^{25}$ In this regard, studies have demonstrated that treatment with ghrelin in a rat model of endotoxemia significantly decreased circulating levels of cytokines. ${ }^{26}$ Based on this knowledge, we sought to determine whether ghrelin can alter cytokine levels in CLP-induced sepsis. Our study showed that intravenous administration of ghrelin starting at 5 hours after CLP for 15 hours, markedly reduced both TNF- $\alpha$ and interleukin (IL)6 plasma and peritoneal fluid levels. ${ }^{27}$ These studies provided preclinical evidence that ghrelin is a beneficial therapeutic agent for sepsis.

\section{Mechanism of action of ghrelin in sepsis}

Although the beneficial role of ghrelin in downregulating cytokines was reported in several studies, ${ }^{24,26,27}$ the mechanism of action of ghrelin in sepsis has only been unraveled within the last few years. In this regard, whether the beneficial effect of ghrelin is mediated by its receptors on macrophages (Kupffer cells and peritoneal macrophages) was explored by us. ${ }^{27}$ To test the hypothesis, we treated Kupffer cells and peritoneal macrophages with lipopolysaccharide (LPS), a potent stimulator of cytokine production. When these cells were treated with LPS alone, as expected, a dramatic increase in TNF- $\alpha$ and IL- 6 levels were observed. In contrast, when these cells were co-incubated with ghrelin and LPS, TNF- $\alpha$ and 


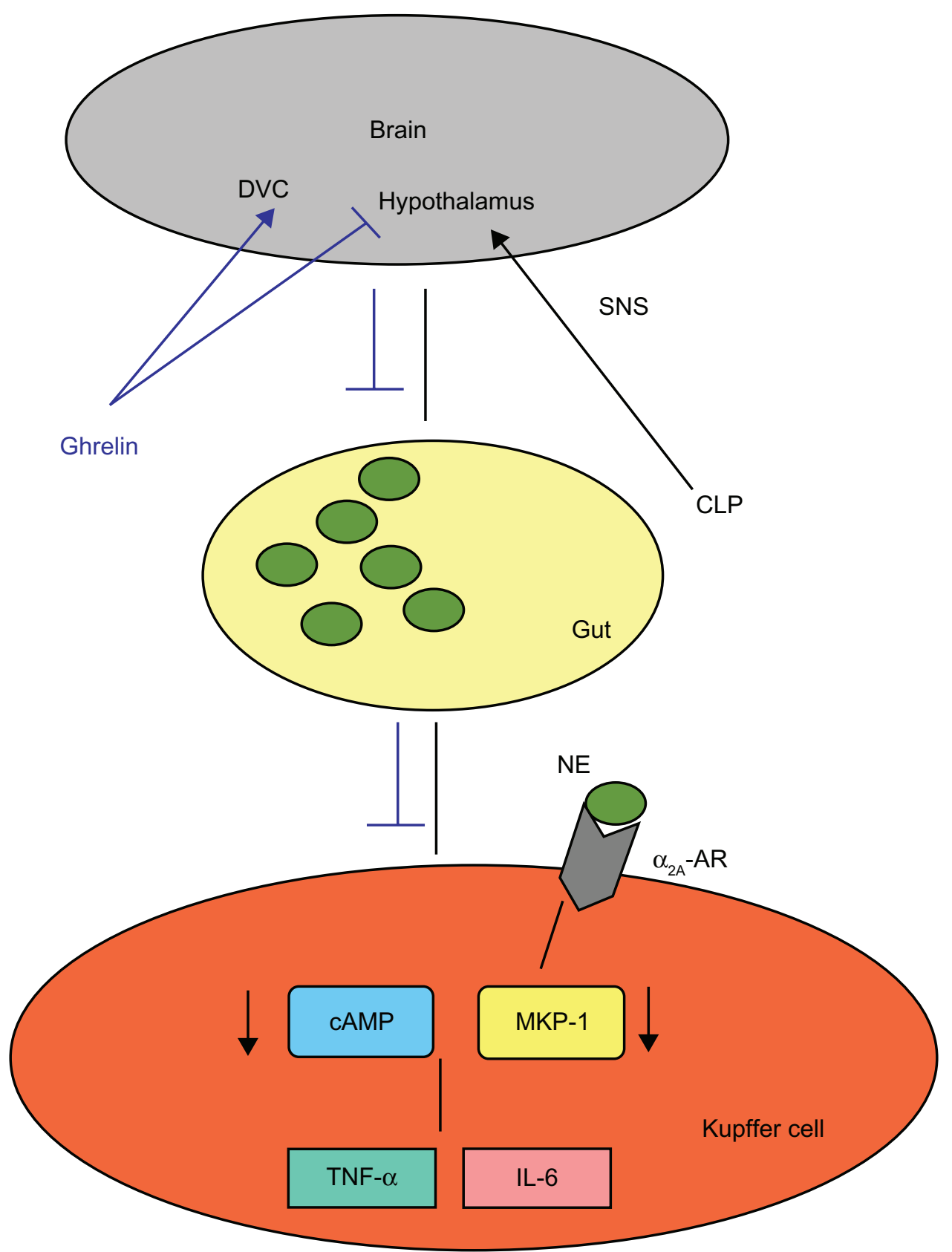

Figure I Polymicrobial sepsis induced by cecal ligation and puncture (CLP) activates the sympathetic nervous system (SNS) and causes the release of norepinephrine (NE) from the sympathetic fibers in the gut. The NE then enters into the circulation and travels through the portal vein into the liver. While in the liver, NE binds to the $\alpha_{2 A}$-adrenoceptors $\left(\alpha_{2 A}-\mathrm{AR}\right)$ and activates the signaling pathway(s) responsible for the production and release of TNF- $\alpha$, IL- 6 and other pro-inflammatory cytokines from Kupffer cells and thereby produces hepatic dysfunction. Ghrelin, a stomach-derived peptide, reaches the dorsal vagal complex (DVC) in the brain by crossing the blood-brain barrier, stimulates GHSRla receptors, activates the vagus nerve and in turn, through the cholinergic pathways, downregulates TNF- $\alpha$ and IL- 6 release from macrophages such as Kupffer cells in the liver possibly by increasing cAMP levels and restoring MKP-I. While activating the cholinergic pathway, ghrelin can inhibit the SNS, decrease the release of the sympathetic neurotransmitter, NE, and cause the downregulation of TNF- $\alpha$ and IL-6 from the macrophages. Therefore, ghrelin's beneficial effect in sepsis by inhibiting the TNF- $\alpha$ and IL- 6 release from Kupffer cells in the liver is mediated by the concerted efforts of the sympathetic and the parasympathetic nervous systems.

IL-6 levels remained as high as LPS alone treated samples. This study show that ghrelin's beneficial effect in decreasing cytokine release in sepsis may not be directly mediated by its receptors on the macrophages.

The hepatic dysfunction in sepsis caused by the upregulation of cytokines is, in part, due to the increased sympathetic activation leading to the release of $\mathrm{NE}$ from the gut during sepsis. ${ }^{10}$ Therefore, we hypothesized that the regulation of the sympathetic nervous system possibly by ghrelin can decrease the sympathetic activation, thereby inhibits cytokine release during sepsis. ${ }^{28}$ As expected, sepsis produced a significant increase in plasma NE and TNF- $\alpha$ levels 
at 2 hours after CLP. Intravenous administration of ghrelin markedly decreased these levels at 2 hours after CLP while administration of ghrelin in sham animals did not show any significant changes in either NE or TNF- $\alpha$ levels in plasma. In addition, intracerebroventricular (ICV) administration of ghrelin markedly decreased circulating levels of NE at 2 hours after CLP. Ghrelin's inhibitory effect on plasma NE levels was completely abolished by ICV injection of the GHSR-1a antagonist. Interestingly, ghrelin's inhibition on TNF- $\alpha$ levels was only partially blocked by ICV injection of GHSR-1a antagonist. Furthermore, co-administration with NE partially blocked ghrelin's inhibitory effect on plasma TNF- $\alpha$ levels as well. Ghrelin treatment in septic rats significantly improved MKP-1 mRNA and protein levels from the vehicle group suggesting the inhibitory effect of ghrelin on TNF- $\alpha$ in sepsis may be mediated by MKP-1. ${ }^{15}$ These studies show that ghrelin's beneficial effect in downregulating TNF- $\alpha$ in sepsis is at least in part mediated by the inhibition of the sympathetic nerve activation.

A large body of evidence indicated that the physiological function of ghrelin is mediated by the central and peripheral receptor distributions and that the wide array of GHSR presence suggests diverse pathways for ghrelin action. ${ }^{29}$ One of these studies demonstrates the presence of GHSR in afferent neurons of nodose ganglia, suggesting that ghrelin signals are transmitted to the brain by the vagal afferent nerves. ${ }^{30}$ It has also been shown that central administration of ghrelin stimulates the vagal efferent nerve in anesthetized rats. ${ }^{31}$ These studies suggest an important role for the vagus nerve in ghrelin's physiological functions. Recent studies demonstrated that electrical stimulation of the vagus nerve subsequent to LPS administration in rats prevented the release of TNF- $\alpha$ from macrophages. ${ }^{32-34}$

To determine if the vagus nerve plays any role in the beneficial effect of ghrelin in sepsis, the effect of ghrelin on TNF- $\alpha$ and IL-6 production in septic animals that underwent subdiaphragmatic vagotomy 5 hours after CLP were investigated. ${ }^{27}$ Ghrelin administration immediately following vagotomy completely prevented the inhibitory effect of ghrelin on the circulating levels of TNF- $\alpha$ and IL-6 during late sepsis. In contrast, ghrelin treatment in animals which did not undergo vagotomy exhibited the anti-inflammatory effect of ghrelin during late sepsis. In addition, vagotomy completely prevented the inhibitory effect of ghrelin on the peritoneal fluid levels of TNF- $\alpha$ and IL-6 during late sepsis as well. However, ghrelin treatment in non-vagotomized rats significantly decreased peritoneal fluid levels of these cytokines during late sepsis. Vagotomy also abrogated ghrelin's protective effect on sepsis-induced organ function parameters such as aspartate aminotransferase (AST), alanine aminotransferase (ALT) and lactate. These studies clearly indicated that ghrelin's beneficial effect in sepsis is mediated by the vagus nerve. These studies collectively demonstrated that ghrelin's beneficial effect in inhibiting cytokine release in sepsis is mediated by a combined action of the activation of the vagus nerve and the inhibition of the sympathetic nervous system (Figure 1).

\section{Mechanism of action of ghrelin in age-related inflammation in sepsis}

It is well recognized that the morbidity and mortality associated with sepsis increases with age. It has also been thought that there is a decline in immune function in the elderly, leading to an inadequate inflammatory response which results in increased morbidity and mortality. ${ }^{35}$ However, the impairment of immune function in aging could be due to an uncontrolled inflammatory response leading to excess production of pro-inflammatory cytokines such as TNF- $\alpha$ and IL-6. This notion is supported by our recent studies ${ }^{36}$ showing that aging exacerbates the pro-inflammatory response and worsens tissue injury in endotoxemia. We also examined whether ghrelin has any role in the hyperinflammatory state observed in septic aged rats. Our studies show that while basal plasma ghrelin levels were significantly elevated in the aged rats, endotoxemia produced greater decrease in ghrelin in aged rats as compared to young rats. Similarly, the gene and protein expressions of the ghrelin receptor were significantly decreased in the dorsal vagal complex of aged rats as compared to young rats. Surprisingly, administration of ghrelin failed to protect the aged rats from endotoxemia.

Ghrelin was originally reported to induce growth hormone (GH) release through the pituitary GHSR-1a stimulation. ${ }^{17-19}$ In humans, $\mathrm{GH}$ levels decline approximately $15 \%$ per decade after age $25 .{ }^{37}$ The decline in $\mathrm{GH}$ with age is associated with many adverse effects that occur with aging. ${ }^{37,38}$ Based on this information, we examined if $\mathrm{GH}$ levels were altered in aged rats and, more importantly, whether GH levels have any role in the hyperinflammatory state observed in the aged rats with endotoxemia. Our results indicated that plasma GH levels were markedly decreased in aged rats as compared to young rats. ${ }^{36}$ Interestingly, when these rats were treated with $\mathrm{GH}$, the ghrelin receptor gene and protein expressions in the dorsal vagal complex increased significantly in aged rats. ${ }^{36}$ However, the GH treatment did not alter either the cytokine levels or the organ injury markers in the endotoxemic aged rats ${ }^{36}$ suggesting GH alone is not sufficient to prevent the hyperinflammatory state in aged rats. In contrast, coadministration 
of ghrelin and GH significantly decreased the inflammatory response and organ injury in the aged rats ${ }^{36}$ indicating that ghrelin's hyporesponsiveness due to a decrease in growth hormone contributes to the aged related hyperinflammatory state in sepsis.

\section{Mechanism of action of ghrelin in radiation combined injury $(\mathrm{RCl})$}

We have recently shown that plasma ghrelin levels were markedly decreased in a rat model of radiation combined injury (RCI), polymicrobial sepsis induced by CLP in combination with whole body radiation. ${ }^{39}$ Intravenous administration of ghrelin in RCI markedly reduced plasma NE levels as compared to RCI rats treated with vehicle. ${ }^{39}$ In addition, ghrelin treatment in RCI produced significant decrease in plasma TNF- $\alpha$ and IL-6, gut tissue levels of TNF- $\alpha$ and IL-6 and myeloperoxidase (MPO) activities of the gut, lungs and kidneys demonstrating that ghrelin is indeed beneficial in downregulating the inflammatory response induced by RCI. ${ }^{39}$ This indicates that ghrelin's protective effect in RCI is mediated by the sympathetic nerve activation.

To determine if ghrelin's effect in RCI is also mediated by the activation of the vagus nerve, vagotomized RCI rats were examined for the inflammatory responses and organ injury parameters. Our results indicated that both non-vagotomized and vagotomized RCI rats exhibited increased circulating levels of inflammatory cytokines and organ injury markers compared to sham operated animals. ${ }^{39}$ Likewise, tissue MPO levels were also significantly increased. In contrast, ghrelin treatment in non-vagotomized RCI rats produced markedly reduced levels of these inflammatory parameters. ${ }^{39}$ However, ghrelin treatment did not reduce these levels in vagotomized RCI rats. This study indicated that ghrelin's beneficial effect in RCI is mediated by the vagus nerve. These studies collectively suggest the protective effect of ghrelin in RCI is to be attributed to balancing of the dysregulated sympathetic and parasympathetic nervous system caused by the injuries.

\section{Future studies and perspectives}

Understanding the pathophysiology of sepsis as it relates to the liver is extremely crucial because the liver is believed to be the primary organ responsible for the multi-organ failure in sepsis. In this regard, we have already demonstrated that even at the early stage of sepsis, hepatic function is depressed due to the upregulation of cytokine production from the liver caused by the increased release of NE from the gut. Therefore, therapy towards sepsis should be directed even at the early stages of sepsis when cardiovascular response appears to be rather normal. Since ghrelin is able to inhibit the early rise in NE levels, ghrelin could be such a candidate for therapy.

In addition, it is also well recognized that vagus nerve stimulation could be beneficial in sepsis. Due to the fact that ghrelin activates the vagus nerve, ghrelin can potentially provide the benefit caused by the vagus nerve stimulation. On the other hand, ghrelin can inhibit the sympathetic nerve activation causing the decrease in NE release and subsequent inhibition of the pro-inflammatory cytokines from the liver during sepsis.

Although our in vitro studies strongly suggest that ghrelin's inhibitory effect on pro-inflammatory cytokine release from the liver and the peritoneal macrophages is not mediated by ghrelin receptors on macrophages, it is not known if ghrelin can protect against the increase in pro-inflammatory cytokines caused by sepsis mediators other than NE. Since ghrelin decreases the mortality of septic animals, it is plausible that ghrelin can be protective against other mediators. Future studies are needed for such a concept.

In aging, we show that ghrelin's hyporesponsiveness attributes to the hyperinflammatory state observed in sepsis and that GH upregulates the ghrelin receptors in the dorsal vagal complex and thus increase the activity of ghrelin. We also show here ghrelin is beneficial in RCI, an experimental model to mimic clinical situation related to the terrorist radiation exposure scenario. In RCI, the protective effect of ghrelin is balancing the dysregulated sympathetic/parasympathetic nervous system caused by the injuries. Future studies are needed to determine whether ghrelin can be beneficial in other acute inflammatory conditions such as hemorrhagic shock and ischemia/reperfusion injury. Nevertheless, the data reviewed herein strongly indicate that ghrelin can be further developed as a potential therapy for sepsis.

\section{Acknowledgements/Disclosures}

This work is supported by the National Institutes of Health grants, R01 GM053008, R01 AG 028352 and R21 AI 080536 (P.W.). The authors report no conflicts of interest.

\section{References}

1. Angus DC, Linde-Zwirble WT, Lidicker J, Clermont G, Carcillo J, Pinsky MR. Epidemiology of severe sepsis in the United States: analysis of incidence, outcome, and associated costs of care. Crit Care Med. 2001;29(7):1303-1310.

2. Annane D, Aegerter P, Jars-Guincestre MC, Guidet B. Current epidemiology of septic shock: the CUB-Rea Network. Am J Respir Crit Care Med. 2003;168(2):165-172.

3. Wang $\mathrm{P}$, Chaudry $\mathrm{IH}$. Mechanism of hepatocellular dysfunction during hyperdynamic sepsis. Am J Physiol. 1996;270(5 Pt 2):R927-R938. 
4. Wang P, Ba ZF, Chaudry IH. Hepatic extraction of indocyanine green is depressed early in sepsis despite increased hepatic blood flow and cardiac output. Arch Surg. 1991;126(2):219-224.

5. Wang P, Ba ZF, Chaudry IH. Hepatocellular dysfunction occurs earlier than the onset of hyperdynamic circulation during sepsis. Shock. 1995;3(1):21-26.

6. Wang P, Ba ZF, Chaudry IH. Mechanism of hepatocellular dysfunction during early sepsis. Key role of increased gene expression and release of proinflammatory cytokines tumor necrosis factor and interleukin-6. Arch Surg. 1997;132(4):364-369; discussion 369-370.

7. Ertel W, Morrison MH, Wang P, Ba ZF, Ayala A, Chaudry IH. The complex pattern of cytokines in sepsis. Association between prostaglandins, cachectin, and interleukins. Ann Surg. 1991;214(2):141-148.

8. Hahn PY, Wang P, Tait SM, Ba ZF, Reich SS, Chaudry IH. Sustained elevation in circulating catecholamine levels during polymicrobial sepsis. Shock. 1995;4(4):269-273.

9. Kovarik MF, Jones SB, Romano FD. Plasma catecholamines following cecal ligation and puncture in the rat. Circ Shock. 1987;22(4):281-290.

10. Yang S, Koo DJ, Zhou M, Chaudry IH, Wang P. Gut-derived norepinephrine plays a critical role in producing hepatocellular dysfunction during early sepsis. Am J Physiol Gastrointest Liver Physiol. 2000;279(6): G1274-G1281.

11. Eisenhofer G, Aneman A, Hooper D, Holmes C, Goldstein DS, Friberg P. Production and metabolism of dopamine and norepinephrine in mesenteric organs and liver of swine. Am J Physiol. 1995;268(4 Pt 1): G641-G649.

12. Eisenhofer G, Aneman A, Hooper D, Rundqvist B, Friberg P. Mesenteric organ production, hepatic metabolism, and renal elimination of norepinephrine and its metabolites in humans. $J$ Neurochem. 1996;66(4):1565-1573.

13. Zhou M, Yang S, Koo DJ, Ornan DA, Chaudry IH, Wang P. The role of Kupffer cell alpha(2)-adrenoceptors in norepinephrine-induced TNFalpha production. Biochim Biophys Acta. 2001;1537(1):49-57.

14. Miksa M, Das P, Zhou M, et al. Pivotal role of the alpha(2A)adrenoceptor in producing inflammation and organ injury in a rat model of sepsis. PLoS One. 2009;4(5):e5504.

15. Jacob A, Rajan D, Pathickal B, et al. The inhibitory effect of ghrelin on sepsis-induced inflammation is mediated by the MAPK phosphatase-1. Int J Mol Med. 2010;25(1):159-164.

16. Kojima M, Hosoda H, Date Y, Nakazato M, Matsuo H, Kangawa K. Ghrelin is a growth-hormone-releasing acylated peptide from stomach. Nature. 1999;402(6762):656-660.

17. Arvat E, Di Vito L, Broglio F, et al. Preliminary evidence that Ghrelin, the natural GH secretagogue (GHS)-receptor ligand, strongly stimulates GH secretion in humans. $J$ Endocrinol Invest. 2000;23(8):493-495.

18. Date Y, Murakami N, Kojima M, et al. Central effects of a novel acylated peptide, ghrelin, on growth hormone release in rats. Biochem Biophys Res Commun. 2000;275(2):477-480.

19. Nass R, Toogood AA, Hellmann P, et al. Intracerebroventricular administration of the rat growth hormone $(\mathrm{GH})$ receptor antagonist $\mathrm{G} 118 \mathrm{R}$ stimulates GH secretion: evidence for the existence of short loop negative feedback of GH. J Neuroendocrinol. 2000;12(12):1194-1199.

20. Wiley KE, Davenport AP. Comparison of vasodilators in human internal mammary artery: ghrelin is a potent physiological antagonist of endothelin-1. Br J Pharmacol. 2002;136(8):1146-1152.
21. Nagaya N, Kojima M, Uematsu M, et al. Hemodynamic and hormonal effects of human ghrelin in healthy volunteers. Am J Physiol Regul Integr Comp Physiol. 2001;280(5):R1483-R1487.

22. Wu R, Zhou M, Cui X, Simms HH, Wang P. Upregulation of cardiovascular ghrelin receptor occurs in the hyperdynamic phase of sepsis. Am J Physiol Heart Circ Physiol. 2004;287(3):H1296-H1302.

23. Basa NR, Wang L, Arteaga JR, Heber D, Livingston EH, Tache Y. Bacterial lipopolysaccharide shifts fasted plasma ghrelin to postprandial levels in rats. Neurosci Lett. 2003;343(1):25-28.

24. Li WG, Gavrila D, Liu X, et al. Ghrelin inhibits proinflammatory responses and nuclear factor-kappaB activation in human endothelial cells. Circulation. 2004;109(18):2221-2226.

25. Koo DJ, Chaudry IH, Wang P. Kupffer cells are responsible for producing inflammatory cytokines and hepatocellular dysfunction during early sepsis. J Surg Res. 1999;83(2):151-157.

26. Chang L, Zhao J, Yang J, Zhang Z, Du J, Tang C. Therapeutic effects of ghrelin on endotoxic shock in rats. Eur J Pharmacol. 2003;473(2-3): 171-176.

27. Wu R, Dong W, Cui X, et al. Ghrelin down-regulates proinflammatory cytokines in sepsis through activation of the vagus nerve. Ann Surg. 2007;245(3):480-486.

28. Wu R, Zhou M, Das P, et al. Ghrelin inhibits sympathetic nervous activity in sepsis. Am J Physiol Endocrinol Metab. 2007;293(6): E1697-E1702.

29. Cowley MA, Grove KL. Ghrelin - satisfying a hunger for the mechanism. Endocrinology. 2004;145(6):2604-2606.

30. Date Y, Murakami N, Toshinai K, et al. The role of the gastric afferent vagal nerve in ghrelin-induced feeding and growth hormone secretion in rats. Gastroenterology. 2002;123(4):1120-1128.

31. Sato N, Kanai S, Takano S, Kurosawa M, Funakoshi A, Miyasaka K. Central administration of ghrelin stimulates pancreatic exocrine secretion via the vagus in conscious rats. Jpn J Physiol. 2003;53(6):443-449.

32. Tracey KJ. The inflammatory reflex. Nature. 2002;420(6917):853-859.

33. Wang H, Yu M, Ochani M, et al. Nicotinic acetylcholine receptor alpha7 subunit is an essential regulator of inflammation. Nature. 2003;421(6921):384-388.

34. Borovikova LV, Ivanova S, Zhang M, et al. Vagus nerve stimulation attenuates the systemic inflammatory response to endotoxin. Nature. 2000;405(6785):458-462.

35. Renshaw M, Rockwell J, Engleman C, Gewirtz A, Katz J, Sambhara S. Cutting edge: impaired Toll-like receptor expression and function in aging. J Immunol. 2002;169(9):4697-4701.

36. Wu R, Zhou M, Dong W, et al. Ghrelin hyporesponsiveness contributes to age-related hyperinflammation in septic shock. Ann Surg. 2009;250(1):126-133.

37. Sherlock M, Toogood AA. Aging and the growth hormone/insulin like growth factor-I axis. Pituitary. 2007;10(2):189-203.

38. Nass R, Park J, Thorner MO. Growth hormone supplementation in the elderly. Endocrinol Metab Clin North Am. 2007;36(1):233-245.

39. Shah KG, Wu R, Jacob A, et al. Human ghrelin ameliorates organ injury and improves survival after radiation injury combined with severe sepsis. Mol Med. 2009;15(11-12):407-414.
Hepatic Medicine: Evidence and Research

\section{Publish your work in this journal}

Hepatic Medicine: Evidence and Research is an international, peerreviewed, open access journal covering all aspects of adult and pediatric hepatology in the clinic and laboratory including the following topics: Pathology, pathophysiology of hepatic disease; Investigation and treatment of hepatic disease; Pharmacology of drugs used for the treatment of hepatic disease. Issues of patient safety and quality of care will also be considered. The manuscript management system is completely online and includes a very quick and fair peer-review system, which is all easy to use. Visit http://www.dovepress.com/ testimonials.php to read real quotes from published authors. 